\title{
LÍNGUAS EM CONFLITO: MODELOS DE ACESSO LEXICAL A PARTIR DO INPUT ORTOGRÁFICO EM BILIINGUES E MULTILÍNGUES E O EFEITO DO MULTILINGUISMO SOBRE AS FUNÇÕES EXECUTIVAS
}

\author{
Marina Fernandes Neves Lameira ${ }^{1}$ \\ Elaine Cristina de Barros Torresi ${ }^{1}$ \\ Katerina Lukasova ${ }^{1}$ \\ Maria Teresa Carthery-Goulart ${ }^{1}$ \\ ㄴUniversidade Federal do ABC, São Bernardo do Campo, São Paulo, Brasil
}

Resumo: O fenômeno do bilinguismo é a premissa essencial da tradução. Este trabalho apresenta modelos cognitivos contemporâneos que explicam o acesso lexical de bilíngues e multilíngues para o reconhecimento de palavras escritas, a partir de experimentos com homógrafos interlinguais. São abordados os trabalhos sobre conflito entre duas ou mais línguas,e como seus achados convergem para a hipótese de acesso não-seletivo ao léxico, o que elucida o impacto do bilinguismo nos processos de leitura e tradução. Como objetivo secundário, e a partir de uma perspectiva neurocientífica, também descrevemos as mudanças no cérebro e nos processos mentais decorrentes à experiência do bilinguismo e do multilinguismo. Os estudos mostram vantagens nas funções executivas (atualização, inibição e flexibilidade mental) e mudanças estruturais e funcionais em circuitos, especialmente envolvendo os córtices pré-frontais bilateralmente. Estas mudanças têm sido apontadas como fatores importantes de proteção (reserva cognitiva e cerebral) frente a diferentes situações de perdaneural.

Palavras-chave: Homógrafos Interlinguais; Multilinguismo; Conflito Linguístico; Funções Executivas 


\title{
LANGUAGES IN CONFLICT: MODELS FOR LEXICAL ACCESS BASED ON ORTOGRAPHIC INPUT IN BILINGUALS OR MULTILINGUALS AND THE EFFECT OF MULTILINGUALISM IN THE EXECUTIVE FUNCTIONS
}

\begin{abstract}
The phenomenon of bilingualism is the essential premise for translation. This paper presents contemporary cognitive models for written word recognition, based on previous experimental work with interlingual homographs. We will discuss studies on language conflict between two or more languages, and how these findings provide support for the language non-selective hypothesis for lexical access, which elucidates the impact of bilingualism in the processes of reading and translation. As a secondary goal and with a neuroscientific perspective, we will also describe the changes in the brain and mental processes that underlie the experience of bilingualism and multilingualism. Studies discussed here demonstrate an advantage in executive function (updating, inhibition and mental flexibility) as well as structural and functional changes in circuits, especially involving the prefrontal cortices bilaterally. These changes have been indicated as important protective factors in different situations of neural loss.
\end{abstract}

Keywords: Interlingual Homographs; Multilingualism; Language Conflict; Executive Function

\section{Introdução}

O domínio de uma ou mais língua, além da materna, tem se tornado altamente desejável após a revolução técnico-científica e informacional e a ampliação do acesso à internet. A este respeito, numa palestra dada em 2013, Gregg Roberts, pesquisador em educação multilíngue, fez a asserção: "O monolinguismo é o analfabetismo do século XXI". O estabelecimento do Quadro Comum Europeu de Referência para Línguas $(1989,1996)$, que define as habilidades linguísticas a serem desenvolvidas para o domínio de línguas estrangeiras, oficializou a importância do multilinguismo no mundo globalizado.

O bilinguismo como regra e não exceção no mundo globalizado recebe a atenção de linguistas, educadores e neurocientistas, que 
têm se ocupado, por exemplo, em investigar a arquitetura neural subjacente às línguas dos bilíngues, os processos de seleção bilíngue e/ou alternância entre línguas de acordo com a necessidade comunicativa. A relação entre línguas no cérebro envolve processos complexos, que podem resultar na disputa por recursos cognitivos. Hernández compara as línguas de um falante bilíngue a duas espécies vivendo dentro de um mesmo ecossistema: dependendo dos recursos existentes, elas podem cooperar ou competir entre si

O presente trabalho tem como objetivo principal apresentar modelos cognitivos contemporâneos que explicam o acesso lexical de bilíngues e multilíngues para a leitura, a partir de experimentos com cognatos e homógrafos interlinguais. Abordaremos também os trabalhos sobre conflito entre duas ou mais línguas, e como seus achados convergem para a hipótese de acesso nãoseletivo ao léxico, que elucida o impacto do bilinguismo nos processos de leitura e tradução, contribuindo assim com o tema principal desse número. Como objetivo secundário, e a partir de uma perspectiva neurocientífica, neste trabalho também descreveremos as mudanças no cérebro e nos processos mentais decorrentes da experiência do bilinguismo e do multilinguismo. Os estudos mostram vantagens nas funções executivas (atualização, inibição e flexibilidade mental) e mudanças estruturais e funcionais em circuitos, especialmente envolvendo os córtices pré-frontais bilateralmente. Como veremos, essas mudanças têm sido apontadas como fatores importantes de proteção (reserva cognitiva e cerebral) frente a diferentes situações de perda neural.

O texto está organizado em três sessões. Na primeira, vamos abordar as diferentes definições para o bilinguismo e multilinguismo e o modelo de Jessner, que mostra o dinamismo da experiência bilíngue/ multilíngue. Na segunda, apresentaremos os debates em relação aos processos de acesso lexical bilíngue e apresentaremos o modelo BIA+ e sua versão mais atual, o Multilink. Na terceira, falaremos dos processos cognitivos envolvidos no manejo de duas ou mais línguas e como isso pode resultar no desenvolvimento de proteção cognitiva. 


\section{Bilinguismo e Multilinguismo: aspectos gerais e definições}

Informações sobre o número de bilíngues na população mundial são controversas, mas considerando que temos cerca de 7000 línguas distribuídas em apenas 193 países, é razoável supormos que mais da metade da população mundial é bilíngue (Associated Press; European Commission). O bilinguismo está presente em todos os continentes, em todas as classes sociais e em todas as idades, avançando na medida em que as fronteiras sociais, políticas e econômicas se estreitam e os meios de comunicação evoluem, sendo portanto, uma experiência cognitiva bastante comum que merece ser avaliada com atenção (Marian \& Shook; Lewis, Simon \& Fennig).

No Brasil, segundo um estudo feito por Oliveira, com dados colhidos até 2009, eram falados 200 idiomas, sendo 170 línguas indígenas (autóctones) e 30 línguas alóctones (faladas nas comunidades de descendentes de imigrantes). Assim, apesar de sermos considerados um país monolíngue, devemos reconhecer a extensão da experiência bi e multilíngue no país, a partir dos povos indígenas, das comunidades de imigrantes, dos povos que vivem em regiões de fronteira, do número crescente de alunos de educação bilíngue e da comunidade que utiliza a língua brasileira de sinais (Libras) (Cavalcanti; Preuss \& Álvarez).

A abordagem do multilinguismo pode ser extremamente multifacetada, podendo-se considerar o ponto de vista linguístico, social, psicológico, neurocientífico, entre outros. Isto torna imprescindível que pesquisadores adotem definições de bilinguismo e multiliguismo que caibam no escopo de suas pesquisas. As primeiras tentativas de definição de bilinguismo passaram pelas posteriormente designadas "visão minimalista", que considera bilíngue aquele que é capaz de produzir frases simples numa língua estrangeira (por exemplo "au revoir", "good morning", etc.); e "visão maximalista", que considera bilíngue aquele que possui domínio semelhante ao nativo de duas línguas (discussão em Megale). Entre essas duas definições há muitas outras; no entanto, modernamente entende-se que o bilíngue é o indivíduo 
com capacidade de se comunicar em duas línguas, sem que necessariamente tenha o mesmo grau de proficiência em ambas ou igual desenvoltura nas quatro habilidades linguísticas: compreensão auditiva, compreensão escrita, expressão oral e expressão escrita (Grosjean (a); Megale; Grosjean (b)). Além disso, nas últimas décadas, outros aspectos têm sido considerados fundamentais para definir as populações e contrastá-las entre estudos.

O termo bilinguismo, independentemente da definição utilizada, não se refere, como já dissemos anteriormente, a um fenômeno uniforme; ele está relacionado obrigatoriamente a diferentes padrões de aquisição e uso da língua, que podem potencialmente influenciar o desempenho dos bilíngues nos testes a que são submetidos, nas mudanças estruturais e funcionais do cérebro e na questão da reserva cognitiva.

Hamers e Blanc, ao longo do seu livro Bilinguality and Bilingualism, propõem fazer uma distinção entre bilinguismo e bilingualidade. Bilinguismo seria o fenômeno global que envolve aspectos psicológicos do sujeito, bem como o uso que faz das línguas em relação a si próprio e à sociedade. Já a bilingualidade estaria relacionada apenas aos aspectos psicológicos individuais de quem acessa duas línguas. Para esses autores, os bilíngues poderiam ser classificados em relação a seis dimensões principais, cujas denominações e definições estão expostas no Quadro 1 abaixo.

Quadro 1 - Critério de classificação de bilíngues segundo Hamers e Blanc

\begin{tabular}{|c|c|c|c|}
\hline DIMENSÕES & \multicolumn{2}{|c|}{ DENOMINAÇÃO } & DEFINIÇÃO \\
\hline \multirow{2}{*}{$\begin{array}{l}\text { Competência } \\
\text { Relativa }\end{array}$} & \multicolumn{2}{|c|}{ Balanceado } & Competência na L1=L2 \\
\hline & \multicolumn{2}{|c|}{ Dominante } & Competência na L1 > L2 ou L2>L1 \\
\hline \multirow{2}{*}{$\begin{array}{l}\text { Organização } \\
\text { Cognitiva }\end{array}$} & \multicolumn{2}{|c|}{ Composto } & Uma única representação cognitiva para as duas línguas \\
\hline & \multicolumn{2}{|c|}{ Coordenado } & Duas representações cognitivas, uma para cada língua \\
\hline \multirow{4}{*}{$\begin{array}{l}\text { Idade de } \\
\text { Aquisição }\end{array}$} & \multirow[t]{2}{*}{ Infantil } & Simultâneo & L1 e L2 adquiridas ao mesmo tempo e antes dos 10 anos \\
\hline & & Consecutivo & L2 adquirida depois da L1 e antes dos 10 anos \\
\hline & \multicolumn{2}{|c|}{ Adolescente } & L2 adquirida entre 11 e 17 anos \\
\hline & \multicolumn{2}{|l|}{ Adulto } & L2 adquirida depois dos 17 anos \\
\hline
\end{tabular}

Cad. Trad., Florianópolis, v. 40, $\mathbf{n}^{0}$ esp. 2, p. 185-216, set-dez, 2020. 


\begin{tabular}{|l|l|l|}
\hline Status das & Aditivo & Não há prejuízo da L1 \\
\cline { 3 - 3 } Línguas & Subtrativo & Há perda ou prejuízo da L1 \\
\hline Identidade & Bicultural & Identificação cultural positiva com L1 e L2 \\
\cline { 3 - 3 } & Monocultural & Identidade cultural referente à L1 ou L2 \\
\cline { 3 - 3 } & $\begin{array}{l}\text { Acultural } \\
\text { Descultural }\end{array}$ & Identidade cultural referente apenas à L2 \\
\cline { 3 - 3 } & Sem identidade cultural \\
\hline Exogenidade & Endógeno & Presença da L2 na comunidade \\
\cline { 3 - 3 } & Exógeno & Ausência da L2 na comunidade \\
\hline
\end{tabular}

Fonte: Hamers e Blanc

Podemos considerar ainda, como fizeram Butler e Hakuta e Chin e Wigglesworth, outros fatores, como o contexto de aquisição (na escola ou em família), o grau de bilinguismo e sua relação com as habilidades linguísticas, motivação na aquisição (devido à imigração, uso recreativo, busca de novas oportunidades de trabalho, religião, etc.) ou o até mesmo o fato de a segunda língua passar a ser a língua dominante, estabelecendo assim novas formas de classificação (Quadro 2).

Quadro 2 - Adaptação das formas de classificação de bilíngues proposta por Butler e Hakuta, e Chin e Wigglesworth

\begin{tabular}{|l|l|l|}
\hline DIMENSÕES & DENOMINAÇÃO & DEFINIÇÃO \\
\hline $\begin{array}{l}\text { Grau de } \\
\text { Bilinguismo }\end{array}$ & Passivo & Compreende, mas não produz enunciados em L2 \\
& Ativo & Produz enunciados em L2 \\
& Limitado & Tem conhecimento bastante limitado em uma das línguas \\
\hline $\begin{array}{l}\text { Contexto de } \\
\text { Aquisição }\end{array}$ & Natural & Aprende naturalmente \\
\hline Motivação & Escolar & Aprende a L2 em contexto secundário (formal) \\
\hline $\begin{array}{l}\text { Orientação } \\
\text { Social }\end{array}$ & $\begin{array}{l}\text { Subcunstancial } \\
\text { Diferencial }\end{array}$ & $\begin{array}{l}\text { Teve a oportunidade de escolher aprender a L2 } \\
\text { Pertence a gruppos levados a aprender a L2 }\end{array}$ \\
\hline Idade de & Vaitiverdendo a competência na L1 na medida que aprende a L2 \\
Aquisição & Precoce & Tem desenvolvimento diferenciado na L1 e L2 \\
\hline
\end{tabular}

Fonte: Butler \& Hakuta e Chin \& Wigglesworth

Cad. Trad., Florianópolis, v. 40, $\mathrm{n}^{0}$ esp. 2, p. 185-216, set-dez, 2020. 
Luk e Bialystok, buscando entender os principais fatores envolvidos na experiência bilíngue, aplicaram testes de proficiência em inglês e um questionário linguístico a 110 bilíngues adultos. Avaliando as repostas ao questionário e a performance nos testes, através de análise fatorial, eles puderam extrair dois fatores principais ligados ao bilinguismo: um relacionado à proficiência na língua e o outro ligado ao uso diário das línguas. Observaram ainda que a auto-avaliação (medida subjetiva) da proficiência parece estar forte e positivamente correlacionada com as avaliações objetivas na amostra estudada. Assim, os autores afirmam que o bilinguismo não é uma variável categórica e sugerem que, a priori, além de dividirem os indivíduos em bilíngues e monolíngues, os estudos deveriam caracterizar a amostra bilíngue da maneira mais ampla possível, informando os leitores sobre os principais critérios classificatórios da amostra utilizada, uma vez que eles podem influenciar os resultados observados.

Convergente com essa visão e sumarizando os aspectos mencionados acima, Baker destaca que pesquisas e estudos em Bilinguismo devem, antes de mais nada, distinguir entre bilinguismo societal e individual e considerar as seguintes dimensões do bilinguismo: a) habilidade (competência produtiva $\mathrm{x}$ receptiva); b) domínios de uso (ex: casa, escola, trabalho, etc.); c) proficiência relativa entre línguas (bilinguismo balanceado ou não-balanceado); d) tipo de aquisição (simultânea x consecutiva); e) desenvolvimento (bilinguismo ocorre no início da aquisição da linguagem ou já quando a língua 1 está bem estabelecida); f) culturalidade (biculturalismo, multiculturalismo); g) contexto social (endógeno - uso diário da língua na sociedade x exógeno - imigrantes, estudantes estrangeiros); h) eletividade ou circunstancialidade, ou seja, bilinguismo eletivo (escolha de adquirir outra língua) ou circunstancial (necessidade de aprender outra língua); i) relação de adição ou subtração do bilinguismo, ou seja, bilinguismo aditivo (convivência societal das duas línguas) ou subtrativo (conflito entre elas).

Um outro aspecto fundamental, quando se pensa nas dimensões do bilinguismo, é que não se deve considerar a proficiência 
relativa entre línguas como uma característica definitiva, uma vez que, mesmo depois da fase de aquisição inicial de uma língua, há um desenvolvimento linguístico constante. Neste quesito, o Modelo de Sistemas Dinâmicos do Multilinguismo - DSMM (Jessner), representado na Figura 1, propõe uma visão holística da aquisição de uma ou mais línguas estrangeiras, abordando o fenômeno sob o ponto de vista do desenvolvimento linguístico longitudinal, e adotando o conceito de sistemas dinâmicos da física para comparar a interrelação entre sistemas linguísticos de um falante. A autora deste modelo questiona a visão de teóricos de aquisição da linguagem, que, segundo ela, abordam o desenvolvimento linguístico como um processo linear e estático, em oposição a pesquisadores da linguística aplicada, que, por sua vez, o encaram como complexo e dinâmico.

Considerando uma alta demanda dos sistemas de memória para o armazenamento de conhecimento lexical em múltiplas línguas, sem que ocorressem perdas, Jessner reconhece "um desenvolvimento linguístico não-linear com mudanças contínuas". Nesta perspectiva, isso deve-se à disputa por espaço na representação cognitiva de uma língua ou de outra para suprir necessidades de comunicação do falante, que pode transitar em diferentes contextos linguísticos ao longo de sua vida. Além disso, considera-se que o desenvolvimento das línguas de multilíngues, em analogia aos sistemas dinâmicos, é reversível, o que se comprovaria a partir dos fenômenos de erosão linguística (retração do conhecimento linguístico devido à falta de uso) e de fossilização (estabilização de proficiência num mesmo nível, sem evolução), sendo ambos fenômenos mais frequentes em multilíngues que em bilíngues, devido ao esforço cognitivo requerido para a manutenção de todas as línguas. Devido a esta complexidade, o multilinguismo deveria ser abordado de maneira diferente do bilinguismo. 
Figura 1 - Exemplo esquemático da proficiência nas línguas de um indivíduo multilíngue ao longo da vida

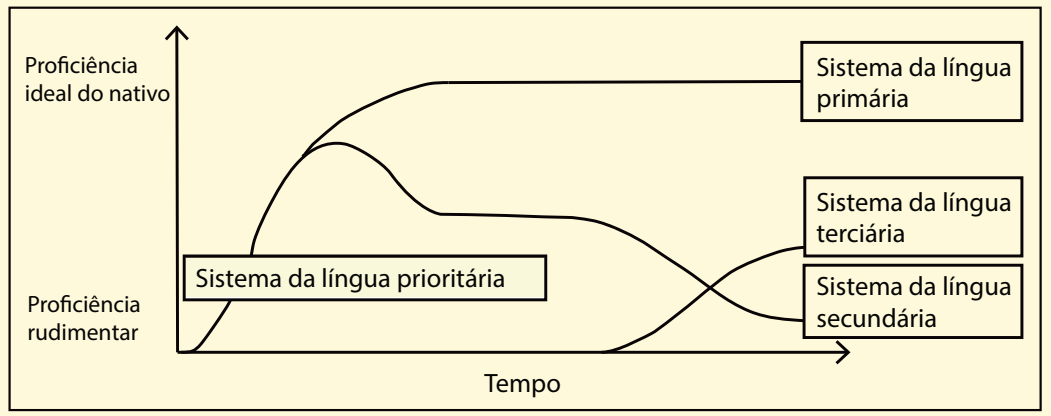

Fonte: Adaptado de Jessner. Nesta figura observa-se a alteração da proficiência de um indivíduo em três línguas ao longo do tempo. Note-se que, entre segunda e a terceira língua, há uma relação de interdependência, uma vez em que a proficiência na segunda língua, ora estável, cai à medida em que a proficiência na terceira língua aumenta, até o momento que a terceira a ultrapassa.

\section{Os processos de acesso lexical bilíngue conforme o modelo de ativação interativa bilíngue BIA +}

O uso das línguas realizado por indivíduos multilíngues é um comportamento observável que permite uma compreensão global do processamento da linguagem. Kroll e Dussias elencam duas questões principais do acesso lexical bilíngue, a saber: a) como se daria a negociação dos limites entre duas línguas que podem, ou não, compartilhar traços e b) quais seriam as representações mentais, processos e mecanismos de aprendizagem que dariam suporte a isto. Entre as propostas para o acesso lexical, temos a visão seletiva das línguas (language selective), na qual o contexto determina qual das línguas é (exclusivamente) acessada (Rodriguez-Fornells et al.), e a visão não seletiva (language non-selective) (De Bruijn et al.; Lemhöfer \& Dijkstra; Kerkhofs et al.; Von Studnitz \& Green; Macizo, Bajo \& Martín; Hsieh et al.), representada, entre outros, pelo modelo BIA + (Bilingual Interactive Model + ) de Dijkstra 
e Van Heuven. O modelo BIA +, representado na Figura 2, propõe regras para a organização do léxico bilíngue quando ativado a partir de input ortográfico, ou seja, durante a leitura. O modelo foi criado a partir de resultados de estudos que apresentavam como principais tarefas a decisão lexical geral (distinguir palavras de uma ou mais línguas de pseudopalavras) e específica (distinguir palavras de uma língua específica de palavras de outra língua ou pseudopalavras), para explicar o reconhecimento de palavras que não estão em contexto de sentença. A partir do input ortográfico (ou seja, de uma palavra escrita), as representações lexicais passam pelo sistema de identificação (figura 2) e pelo esquema da tarefa (figura 3), podendo haver conflito em um destes níveis (respectivamente, baseado em estímulo e baseado em resposta).

Para o $\mathrm{BIA}+$, a apresentação de input ortográfico gera a ativação paralela (co-ativação) de suas representações ortográficas, fonológicas e semânticas correspondentes no nó da L1 (Língua 1) e da L2 (Língua 2), que competem para serem selecionadas. Em outras palavras, o modelo adere à visão não seletiva, uma vez que considera que, mesmo durante a realização de uma tarefa numa língua $\mathrm{X}$, a língua $\mathrm{Y}$ de um bilíngue será também acessada.

Figura 2 - Sistema de identificação do BIA +

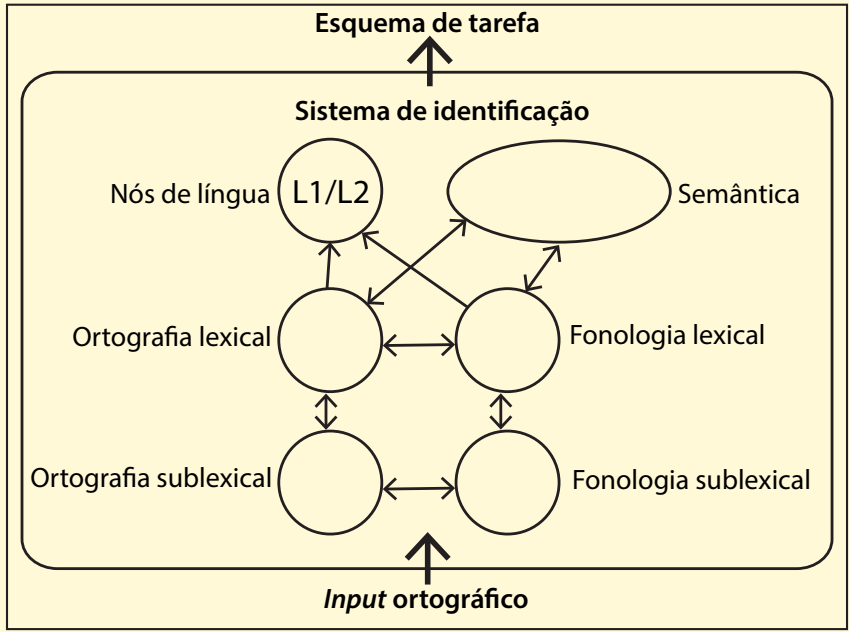

Cad. Trad., Florianópolis, v. 40, n⿳0 esp. 2, p. 185-216, set-dez, 2020. 
Fonte: Adaptada de Dijkstra e Heuven. Esta figura esquematiza o primeiro momento do processamento semântico de acordo com o modelo BIA + . No sistema de identificação, a apresentação de input visual ativa representações nos níveis sublexical e lexical, incluindo aspectos como ortografia e fonologia, em ambos os nós de língua.

Devido à co-ativação, palavras de duas línguas que compartilham traços semânticos, ortográficos e/ou fonológicos, são mais rapidamente ativadas que palavras que não compartilham estes traços. Se estas palavras compartilham a semântica e a ortografia, são cognatos (ex: exemplo: film (inglês - filme) e Film (alemão - filme)). Caso a semântica não seja compartilhada, ou seja, as palavras possuem a mesma ortografia, mas não possuem o mesmo significado, temos homógrafos interlinguais (interlingual homographs; por exemplo: tag (inglês - rótulo) e Tag (alemão dia), também chamados de falsos cognatos. A leitura da palavra “TAG”, para um falante de alemão e inglês, por exemplo, irá ativar simultaneamente as representações ortográficas e semânticas deste item no nó de língua inglesa e nó de língua alemã, levando a um conflito na sua seleção (identificação da palavra como pertencente a uma língua ou outra). Este tipo de conflito é denominado "conflito baseado no estímulo".

O "conflito baseado em resposta", por sua vez, ocorre quando a seleção do item lexical não se dá na língua em que o participante está realizando o teste, ou seja, o participante identifica um item somente como pertencente a uma língua $\mathrm{X}$, mas a tarefa solicita somente a identificação de palavras da língua Y. Este processamento ocorre, segundo o $\mathrm{BIA}+$, no esquema da tarefa, e não mais no sistema de identificação. 
Figura 3 - Esquema da tarefa do BIA+

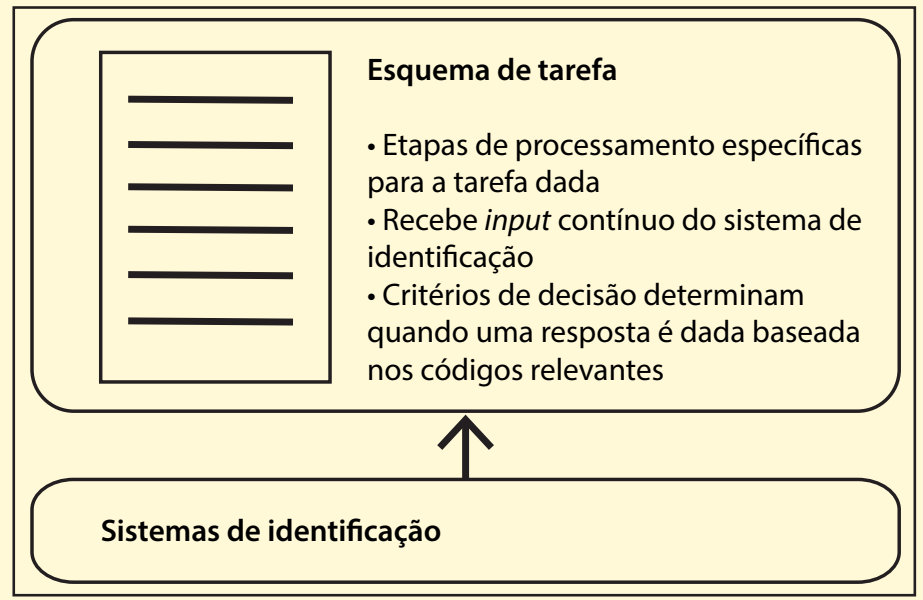

Fonte: Adaptada de Dijkstra e Heuven. Segundo o modelo BIA +, a resposta final do participante ocorre após o processamento do esquema da tarefa. Após a primeira fase de ativação de representações concorrentes no sistema de identificação, o indivíduo tomará uma decisão quanto à seleção de uma destas representações, conforme a tarefa que lhe for imposta.

Os primeiros estudos deste tipo de conflito identificaram diferenças no tempo de reação para a leitura de um homógrafo em tarefas de decisão lexical geral ou específica. Em uma tarefa de decisão lexical geral, o homógrafo interlingual é reconhecido como uma resposta válida em ambas as línguas aceitas, e não há conflito. Na decisão lexical específica, por sua vez, o participante deve responder "sim" se a palavra lida existe em uma língua específica (a língua-alvo da tarefa), ou "não", caso a palavra lida seja considerada como inexistente nesta língua. Caso o homógrafo seja selecionado pelo participante como pertencente à língua não-alvo, haverá um conflito entre a sua seleção e a resposta esperada. A Figura 4 mostra um esquema destes tipos de conflito. 
Figura 4 - Conflito em tarefas de decisão lexical

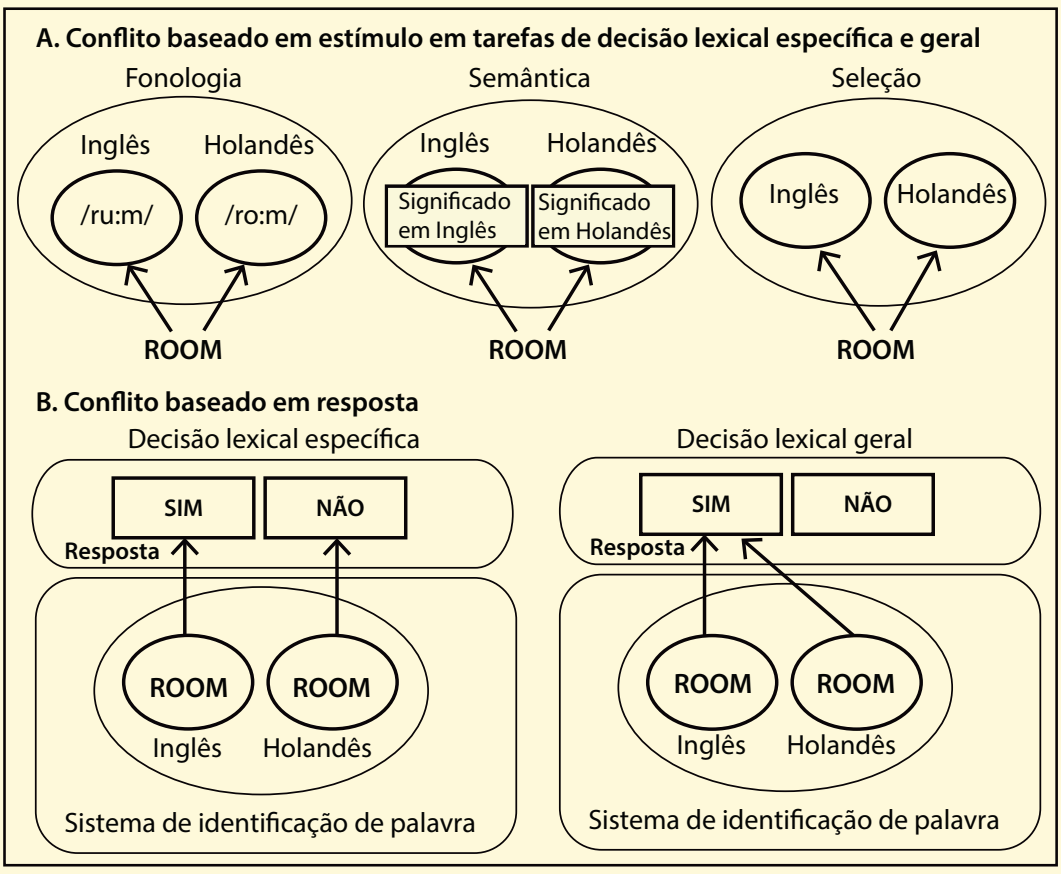

Fonte: Adaptado de Van Heuven e colaboradores. Esta figura demonstra que a leitura de um item lexical pode levar a um conflito devido a mais de uma forma fonológica ou mais de um significado em duas línguas (baseado em estímulo) ou a um conflito na seleção de uma das representações conforme exigências da tarefa (baseado em resposta). Se qualquer uma das línguas for aceita pela tarefa, ambas as representações são aceitáveis, mas se somente uma das línguas for aceita pela tarefa, a seleção de uma das representações constituirá um erro de resposta.

\subsection{Cognatos e homógrafos interlinguais nos estudos sobre} modelos de acesso lexical bilíngue e a proposta do modelo Multilink

De acordo com Lemhöfer e Dijkstra, os cognatos e os homógrafos interlinguais têm sido uma excelente ferramenta para com- 
provação da co-ativação das línguas em estudos de reconhecimento de palavras realizados nas últimas décadas. Os fenômenos de facilitação ou conflito gerados por estas palavras, durante o seu reconhecimento, são tomados como evidência da co-ativação paralela (Lemhöfer \& Dijkstra). Para os cognatos, ocorre o efeito de facilitação, sendo que as palavras cognatas são mais rapidamente reconhecidas que não-cognatas (Lemhöfer, Dijkstra \& Michel).

Para os homógrafos interlinguais pode haver também facilitação, uma vez que seu reconhecimento pode ser mais rápido em tarefas de decisão lexical geral, ou seja, quando ambas as leituras do homógrafo podem ter respostas corretas. No estudo de Lemhöfer, Dijkstra e Michel, foi encontrado um maior efeito de facilitação de cognatos triplos (holandês-inglês-alemão) que cognatos duplos (holandês-alemão), mas mesmo estes foram mais rapidamente reconhecidos que palavras-controle, demonstrando que, em trilíngues, o efeito é o mesmo que aquele encontrado em bilíngues. Por outro lado, pode ocorrer um conflito ou interferência: em tarefas de decisão lexical específica, somente uma das leituras do homógrafo é aceita como resposta correta, e a ativação da leitura concorrente pode levar a aumento no tempo de resposta (Figura 4).

De acordo com BIA +, estes efeitos dependem da similaridade e da frequência das leituras do homógrafo em cada uma das duas línguas: quanto maior a frequência de uma palavra em uma língua, mais chances de sua representação ser selecionada em detrimento da outra, menos frequente. Desta forma, as diferenças nas respostas, por exemplo no tempo de reação ao estímulo de palavras cognatas ou falsas cognatas em duas línguas, evidenciariam interação entre suas representações lexicais, em comparação com palavras-controle (Jouravlev, Lupker \& Jared). Entretanto, achados indicam que outras características linguísticas podem interferir neste fenômeno (Groot).

Van Wijnendaele e Brysbaert conseguiram interferir no processamento da L2 para a L1, com a apresentação de um prime homofônico (um breve estímulo que antecede palavra alvo e compartilha suas características fonéticas) em L2 (holandês) para a identificação 
de um alvo na L1 (francês). Num estudo semelhante, Kerkhofs e colaboradores introduziram um prime que compartilha características semânticas com o alvo, antes da apresentação de um homógrafo interlingual, a fim de facilitar o reconhecimento do mesmo como uma palavra pertencente à L2, e não à L1, numa tarefa de decisão lexical Por exemplo, o homógrafo interlingual "angel”, em inglês, significa anjo e, em holandês, ferrão. Em holandês como L1, ferrão tem frequência mais alta que na L2 inglês (anjo), portanto, segundo o modelo $\mathrm{BIA}+$, a palavra seria identificada como pertencente ao holandês. Entretanto, a introdução do prime, palavra "heaven" (paraíso), afetou o reconhecimento do homógrafo como uma palavra pertencente ao inglês (Figura 5).

Macizo e colaboradores utilizaram o paradigma de priming negativo, visando a induzir um conflito gerado pela ativação da acepção do homógrafo na língua dominante, não-alvo da tarefa. Numa tarefa de julgamento semântico, seus participantes deveriam, na condição crítica do experimento, julgar se o par inglês "toe-pie" estaria associado. Considerando as acepções inglesas, "dedão do pé" e "torta", a resposta deveria ser "não". No entanto, o homógrafo interlingual < pie> que quer dizer "pé” em espanhol (a língua materna dos participantes), segundo o modelo $\mathrm{BIA}+$, deveria gerar uma competição entre ambas as acepções, sendo a espanhola mais rapidamente ativada que seu paralelo $<$ pie $>$ do inglês, pois teria maior frequência de uso no espanhol. Deste modo, para evitar a resposta intuitiva, "sim", e dar a resposta esperada, "não", os participantes tiveram de inibir a acepção espanhola da palavra, incrementando o processamento em uma etapa, e, portanto, demorando mais nas respostas. Os efeitos de interferência foram encontrados também por Schwartz e Fontes durante a apresentação de pares de palavras não relacionadas em inglês (L2), mas relacionadas por meio do espanhol (L1) graças à presença de um homófono (boat-bark). 
Figura 5 - Extensão do BIA+

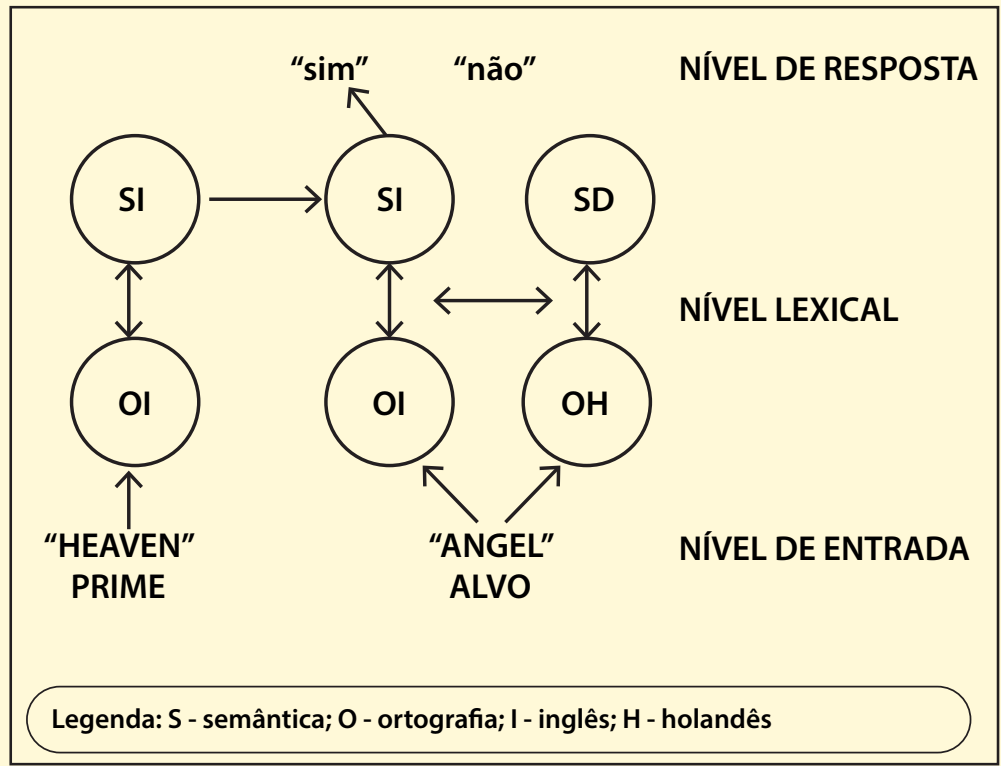

Fonte: Adaptada de Kerkhofs e colaboradores. Nesta figura, o modelo BIA + é extendido à medida em que o esquema de tarefa é manipulado a favor da seleção de uma das representações a partir da introdução de um prime, uma palavra que está semanticamente relacionada com apenas uma das leituras possíveis de um homógrafo.

Resumindo os achados do modelo $\mathrm{BIA}+$, no contexto de pesquisa, assim como aplicado à leitura e/ou a tradução, evidências mostram que se a língua não-alvo for a mais fraca, é menos provável que ela influencie o processamento da língua-alvo, se esta for a mais forte das duas. Este efeito pode, no entanto, ser manipulado a partir da apresentação de um prime, como realizado por Kerkhofs e colaboradores. Analogamente, em condições inversas, a língua mais forte irá influenciar a mais fraca (Groot). Por esta razão, Groot é contrária a tarefas que envolvam as duas línguas, se o objetivo é verificar a influência de uma no processamento da outra. Tarefas bilíngues, necessariamente, ativam o léxico das duas línguas e, portanto, facilitariam sua interação. 
Tomadas em conjunto, descobertas de estudos envolvendo o conflito durante o acesso e a seleção lexical evidenciaram que a interferência pode ocorrer a) tanto da L2 na L1 e vice-versa (Kousaie \& Phillips; Hsieh; et al.); b) por meio de equivalentes de tradução (Macizo; Bajo \& Martín; Thierry \& Wu ((a); (b); (c)) em aprendizes intermediários de L2 (Hsieh et al.; (d)) em jovens adultos e adultos mais velhos (Kousaie \& Phillips); (e)) devido a aspectos fonológicos (Schwartz \& Fontes; Lemhöfer \& Dijkstra; (f)) em contexto de sentença (Elston-Güttler; Gunter \& Kotz; (g)) em línguas logográficas (Hsieh et al.; (h)) apesar de instrução da presença dos homógrafos interlinguais (Von Studnitz \& Green).

Além disso, avanços teóricos tem sido alcançados na busca por um "modelo universal", que preveja a seleção lexical com mais de um léxico e em diferentes tipos de tarefas. Recentemente, com o objetivo de criar um modelo que englobe a seleção lexical tanto em tarefas de produção quanto de compreensão, Dijkstra e colaboradores estão desenvolvendo o modelo Multilink. Atualmente, o modelo é capaz de simular o reconhecimento e a produção de cognatos em tarefas como decisão lexical, nomeação de palavras e tradução oral de palavras. A partir da alimentação do modelo com sistemas lexicais monolíngues ou bilíngues, incluindo informações como leitura fonológica, a frequência de palavras e a similaridade entre vizinhos ortográficos, a inserção de um item lexical como input visual inicial ativa concorrentes de uma ou mais línguas, até que a resposta final do participante seja identificada.

\section{Bilinguismo e Multilinguismo como potencializadores do desenvolvimento das funções executivas e a Reserva Cerebral e Cognitiva}

Estudos mostram que a experiência bilíngue/multilíngue provoca mudanças comportamentais (Bialystok \& Poarch) e na estrutura (Pliatsikas; Moschopoulou \& Saddy) e funcionalidade (Hosoda et $a l$.) do cérebro. As relações entre bi e multilinguismo e plasti- 
cidade cerebral têm justificado e impulsionado pesquisas que se concentram em dois grandes eixos relacionados: os efeitos do bilinguismo sobre as funções executivas (operações relacionadas à atualização, inibição e alternância entre informações) e a reserva cognitiva decorrente dessa experiência.

De modo geral, aceita-se que o termo funções executivas é um constructo relacionado a processos de controle que consistem de uma ampla gama de habilidades, das quais destacam-se, segundo Miyake e colaboradores três ramos principais: inibição, flexibilidade e atualização. A inibição está relacionada ao controle de interferências, seja ele ligado ao autocontrole ou à supressão de informação irrelevante, cujos veículos são a inibição cognitiva e a atenção seletiva, respectivamente. A atualização relaciona-se, por sua vez, com a atualização de informações e o monitoramento, estando ligada, portanto, à memória operacional. Finalmente, a flexibilidade relaciona-se com a capacidade de alteração de estados mentais e alternância entre tarefas.

Figura 6 - Representação das Funções Executivas destacadas por Miyake e colab.

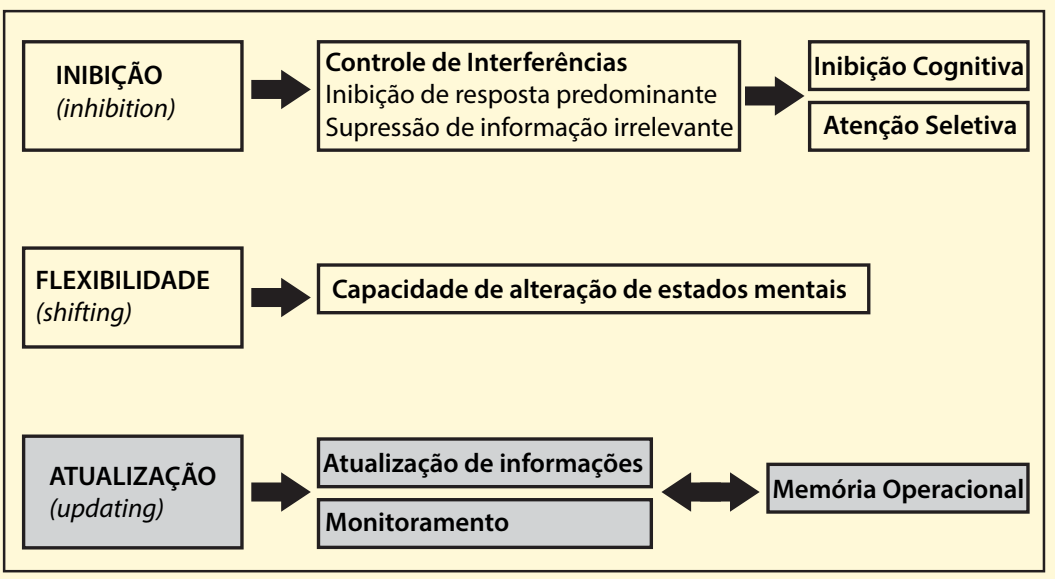

Fonte: Miyake et al.

Esta figura demonstra esquematicamente os três ramos nos quais podem ser subdivididas as funções executivas e as habilidades a elas relacionadas. 
Uma série de estudos associa o bilinguismo com fortalecimento do controle executivo, que se traduz por melhores performances em vários tipos de tarefas, destacando-se os testes de inibição (Blumenfeld \& Marian; Poarch \& Bialystok; Bialystok; Craik \& Luk (a)) e os testes de flexibilidade (Tao; Taft \& Gollan).

Vantagens no controle cognitivo de bilíngues foram encontradas em bebês menores de dois anos (Poulin-Dubois et al.), crianças (Martin-Rhee \& Bialystok; Poarch \& Van Hell), jovens e adultos (Prior \& Macwhinney; Costa; Hernández \& Sebastián-Gallés) e idosos (Bialystok et al. (a)), em bilíngues precoces (Luk: De Sa \& Bialystok) e tardios (Vega-Mendoza) e também em imigrantes (Abreu et al.) e não imigrantes (Friesen et al.). Para evidências contrárias à vantagem cognitiva do bilinguismo, veja De Bruin, Treccani e Della Sala; Paap, Johnson e Sawi; Dunãbeitia e Carreiras; Dunãbeitia et al.; Paap e Greenberg; Paap e Sawi; Mor, Yitzhaki-Amsalem e Prior.

A explicação para essas vantagens estaria no fato de que os bilíngues, diferentemente dos monolíngues, estão constantemente selecionando e monitorando a língua a ser utilizada ao mesmo tempo em que inibem a outra. Essa inibição deve acontecer pois ambas as línguas parecem estar constantemente ativadas, conforme as evidências dos modelos que apresentamos na seção anterior (Dijkstra \& Van Heuven). Como esse mecanismo de seleção e inibição faz parte do sistema de controle executivo, parece natural que este se fortaleça beneficiando a cognição como um todo (Bialystok; Bialystok et al. (b); Kroll; Bobb \& Hoshino).

Todavia, os fatores que afetam a experiência bilíngue, tais como idade de aquisição, proficiência, contexto de aquisição e uso, etc., discutidos na primeira sessão do texto, parecem influenciar os resultados dos estudos (Mohades et al.; Singh \& Mishra; Bialystok et al.; (b); Adesope et al.; Hilchey \& Klein) bem como outras variáveis confundentes como status socioeconômico, inteligência, imigração, genética, educação, estilo de vida e atividades de lazer (De Bruin; Bak \& Della Sala). As pesquisas têm mostrado que mudanças sutis nas condições experimentais, tais como: padrão 
bilíngue da amostra (idade de aquisição, proficiência, padrão de uso das línguas, etc.), tipo de teste e modalidade sensorial utilizados, número de tentativas do teste, entre outras, podem produzir resultados divergentes (Bak (a); (b)). Portanto, a diferença entre populações vai além da língua que elas falam, envolvendo múltiplos aspectos (culturais, sociais, econômicos), o que faz com que os estudos sobre bilinguismo/multilinguismo não sejam algo trivial; Os próximos rumos nas pesquisas devem ser no sentido de determinar como diferentes populações podem produzir diferentes resultados (Bak (a); (b)).

Estudos que associam métodos de neuroimagem e testes de funções executivas, feitos em bilíngues, encontraram diferenças estruturais, funcionais e de conectividade entre o cérebro bilíngue e monolíngue. Wong, Yin e O'Brien apontaram em uma recente revisão que: (1) quanto à estrutura cortical, observa-se maior densidade de substância cinzenta no lobo parietal inferior (envolvido no controle atencional) e maior volume de substância cinzenta no núcleo caudado esquerdo de bilíngues (que tem conexões com o córtex pré-frontal dorsolateral (DLPFC); (2) quanto à conectividade, observa-se que ela é maior entre as redes frontais e parietais em bilíngues que em monolíngues, envolvendo a DLPFC e o lobo parietal inferior e (3) quanto à função, em julgamentos semânticos, bilíngues ativam mais o DLPFC e o córtex frontal inferior que monolíngues.

A teoria da reserva cognitiva explica como, frente a um mesmo grau de alterações cerebrais, indivíduos podem apresentar diferentes níveis de funcionamento cognitivo. Assim, em pessoas com baixa reserva cognitiva (ex. indivíduos analfabetos), graus leves de atrofia cerebral podem já levar a um prejuízo na funcionalidade no dia-a-dia. Por outro lado, em pessoas com maior reserva cognitiva, é necessária uma maior gravidade das alterações estruturais e funcionais cerebrais para que haja grave declínio funcional. A teoria postula que algumas experiências melhoram a capacidade do cérebro de lidar com as perdas e danos, mitigando os efeitos dessas perdas sobre as funções cognitivas (Stern (a); (b)). Vários autores sugerem que o bilinguismo colabora ativamente na formação de 
uma reserva cognitiva, podendo postergar o estabelecimento de demência apresentando portanto efeitos protetivos do sistema nervoso (Craik; Bialystok \& Freedman; Gold; Nathan \& Powell; Bak et al.; Bialystok et al.; (c); Woumans et al.).

Em um dos maiores estudos documentados até o momento sobre reserva cognitiva e bilinguismo, Alladi e colaboradores revisaram os registros de 648 pacientes com demência (sendo 391 bilíngues), comparando a idade de aparecimento dos primeiros sintomas entre os grupos e considerando número de línguas faladas, nível educacional e ocupação. Eles observaram uma latência de 4,5 anos no aparecimento de sintomas em bilíngues tanto para demência por Alzheimer e demência frontotemporal quanto para demência de origem vascular, reproduzindo os achados de Bialystok; Craik; Freedman. Os autores observaram ainda que esse atraso aconteceu até mesmo em pacientes bilíngues e analfabetos.

Em 2011, Luk e colaboradores, usando DTI e MRI (em tarefa de restingstate) puderam observar que bilíngues apresentavam maior conectividade estrutural dos lobos frontais com as regiões adjacentes e posteriores. Eles propuseram que alguns efeitos protetivos (relacionados à reserva cognitiva) observados em bilíngues deviam-se à manutenção da substância branca. Ligado a esse tipo de achado apareceu o termo reserva cerebral, que se refere às alterações estruturais, em termos de volume de substância branca e cinzenta, que resultam de experiências cognitivas importantes, como por exemplo o bilinguismo (Perani \& Abutalebi; Grant; Dennis \& Li; Bialystok e colab. (d)).

Perani e Abutalebi sugerem dois possíveis mecanismos neurais para explicar os efeitos protetivos do bilinguismo: compensação neural (mudanças funcionais) e reserva neural (alterações estruturais), que seriam induzidas pelo aumento da demanda de atenção e controle que o fato de falar duas ou mais línguas exige. Segundo esses autores, o bilinguismo exige forte envolvimento de áreas cerebrais resultando em alterações plásticas das substâncias branca e cinzenta, o que eventualmente poderia proteger o cérebro contra atrofias e neurodegeneração devido ao envelhecimento ou 
doenças. Os autores apontam que as principais diferenças entre o cérebro bilíngue e o monolíngue se concentram em áreas relacionadas ao controle executivo, envolvendo uma rede de regiões no córtex pré-frontal, córtex cingulado anterior, lobo parietal inferior e estruturas subcorticais (caudado e putâmen) e áreas relacionadas à linguagem com regiões no córtex pré-frontal inferior esquerdo e lobo parietal inferior esquerdo.

Segundo Kemmerer, a linguagem influencia e é influenciada pela arquitetura cerebral. A fluidez dos processos neurais de aquisição, tratamento, armazenamento e recuperação da informação que regulam o funcionamento da linguagem são os fatores que respondem pelas alterações que observamos no cérebro a partir do amadurecimento das funções linguísticas. Assim, pela análise dos estudos apresentados até o momento, entendemos que o bilinguismo como vivência linguística é experiência cognitiva ímpar que atua sobre a plasticidade cerebral, modelando e remodelando estruturas, estabelecendo e fortalecendo conexões entre diversas regiões e finalmente contribuindo para a formação de uma reserva cognitiva. Dessa forma, parece-nos coerente propor que a experiência bilíngue pode influenciar também o comportamento nas mais diversas tarefas, principalmente aquelas relacionadas ao controle cognitivo.

\section{Agradecimentos}

Agradecemos à CAPES pelo apoio ao Programa de Pós-Graduação em Neurociência e Cognição da Universidade Federal do ABC. Agradecemos também à FAPESP pelo suporte financeiro às pesquisas (Elaine C. de B. Torresi, número do projeto:2015/09733-6, Marina F. N. Lameira, número do projeto: 2017/00246-0). 


\section{Referências}

Abreu, Pascale MJ Engel de; et al. "Bilingualism enriches the poor: Enhanced cognitive control in low-income minority children”. Psychological science. 23.11, (2012): 1364-1371.

Adesope, Olusola O.; et al. "A systematic review and meta-analysis of the cognitive correlates of bilingualism". Review of Educational Research. 80.2, (2010): 207-245.

Alladi, Suvarna, et al. "Bilingualism delays age at onset of dementia, independent of education and immigration status". Neurology. 81.22, (2013): 1938-1944.

Associated Press. "Some facts about the world's 6,800 tongues". CNN.com/ US. 19.06.2001. Web. 25 mar 2016 < http://edition.cnn.com/2001/US/06/19/ language.glance/index.html?_\%2520s =PM:US $>$.

Bak, Thomas H.; et al. "Does bilingualism influence cognitive aging?". Annals of neurology. 75.6, (2014): 959-963.

Bak, Thomas H. (a). “Beyond a simple 'yes' and 'no””. Cortex. 73, (2015): 332-333.

Bak, Thomas H. (b). "The impact of bilingualism on cognitive ageing and dementia: Finding a path through a forest of confounding variables". Linguistic Approaches to Bilingualism. 6.1-2, (2016): 205-226.

Bialystok, Ellen. "Bilingualism: The good, the bad, and the indifferent". Bilingualism: Language and cognition. 12.1, (2009): 3-11.

Bialystok, Ellen; Craik, Fergus I.M.; Freedman, Morris. "Bilingualism as a protection against the onset of symptoms of dementia". Neuropsychologia. 45.2, (2007): 459-464.

Bialystok, Ellen; Craik, Fergus; Luk, Gigi (a). "Cognitive control and lexical access in younger and older bilinguals". Journal of Experimental Psychology: Learning, memory, and cognition. 34.4, (2008): 859-873. 
Bialystok, Ellen; Craik, Fergus I.M.; Luk, Gigi (b). "Bilingualism: consequences for mind and brain". Trends in cognitive sciences. 16.4, (2012): 240-250.

Bialystok, Ellen; Poarch, Gregory J. "Language experience changes language and cognitive ability". Zeitschrift für Erziehungswissenschaft. 17.3, (2014): 433-446.

Bialystok, Ellen; et al. (a). "Bilingualism, aging, and cognitive control: evidence from the Simon task”. Psychology and aging. 1.9, (2004): 290-303.

Bialystok, Ellen; et al. (b). "Bilingual minds". Psychological science in the public interest. 10.3, (2009): 89-129.

Bialystok, Ellen; et al. (c). "Effects of bilingualism on the age of onset and progression of $\mathrm{MCI}$ and $\mathrm{AD}$ : Evidence from executive function tests". Neuropsychology. 28.2, (2014): 290-304.

Bialystok, Ellen; et al. (d). "Aging in two languages: Implications for public health". Ageing Research Reviews. 27, (2016): 56-60.

Baker, Colin. Foundations of bilingual education and bilingualism. Vol. 79. Multilingual matters, 2011.

Blumenfeld, Henrike K.; Marian, Viorica. "Cognitive control in bilinguals: Advantages in Stimulus-Stimulus inhibition". Bilingualism: Language and Cognition. 17.3, (2014): 610-629.

De Bruijn, Ellen R.A.; et al. "Language context effects on interlingual homograph recognition: evidence from event-related potentials and response times in semantic priming”. Bilingualism: Language and Cognition. 4.2, (2001): 155-168.

De Bruin, Angela; Bak, Thomas H.; Della Sala, Sergio. "Examining the effects of active versus inactive bilingualism on executive control in a carefully matched non-immigrant sample". Journal of Memory and Language. 85, (2015): 15-26.

De Bruin, Angela; Treccani, Barbara; Della Sala, Sergio. "Cognitive advantage in bilingualism: An example of publication bias?". Psychological science. 26.1, (2014): 99-107. 
Butler, Yuko G.; Hakuta, Kenji. "Bilingualism and second language acquisition". The handbook of bilingualism, Bhatia, Tej K.; Ritchie, William C. (Eds.). New York: Blackwell. 2006, pp. 114-144.

Cavalcanti, Marilda C. "Estudos sobre educação bilíngue e escolarização em contextos de minorias linguísticas no Brasil". DELTA: Documentação de Estudos em Lingüística Teórica e Aplicada. 15.SPE, (1999): 385-417.

Chin, Ng Bee; Wigglesworth, Gillian. Bilingualism: An advanced resource book. Nova York: Routledge, 2007.

Craik, Fergus I. M.; Bialystok, Ellen; Freedman, Morris. "Delaying the onset of Alzheimer disease: Bilingualism as a form of cognitive reserve". Neurology. 75.19, (2010): 1729.

Costa, Albert; Hernández, Mireia; Sebastián-Gallés, Núria. "Bilingualism aids conflict resolution: Evidence from the ANT task". Cognition. 106.1, (2008): 59-86.

Dijkstra, Ton; Van Heuven, Walter J.B. "The architecture of the bilingual word recognition system: From identification to decision". Bilingualism: Language and cognition. 5.3, (2002): 175-197.

Dijkstra, Ton; et al. "Multilink: a computational model for bilingual word recognition and word translation”. Bilingualism: Language and Cognition. 22.4, (2018): 657-679.

Duñabeitia, Jon Andoni; et al. "The inhibitory advantage in bilingual children revisited". Experimental psychology. 61.3, (2014): 234-251.

Duñabeitia, Jon Andoni; Carreiras, Manuel. "The bilingual advantage: Acta est fabula". Cortex. 73, (2015): 371-372.

Elston-Güttler, Kerrie E.; Gunter, Thomas C.; Kotz, Sonja A. "Zooming into L2: Global language context and adjustment affect processing of interlingual homographs in sentences”. Cognitive Brain Research. 25.1, (2005): 57-70. 
European Comission- Special Eurobarometer. "Europeans and their languages". Special EUROBAROMETER 243. fev 2006. Web. 25 mar $2016<\mathrm{http} / / /$ ec.europa.eu/public_opinion/archives/ebs/ebs_243_en.pdf $>$.

Friesen, Deanna C.; et al. "Attention during visual search: The benefit of bilingualism”. International Journal of Bilingualism. 19.6, (2015): 693-702.

Gold, Brian T.; Johnson, Nathan F.; Powell, David K. "Lifelong bilingualism contributes to cognitive reserve against white matter integrity declines in aging". Neuropsychologia. 51.13, (2013): 1-16.

Grant, Angela; Dennis, Nancy A.; Li, Ping. "Cognitive control, cognitive reserve, and memory in the aging bilingual brain". Frontiers in psychology. 5, (2014): 1-10.

De Groot, Annette M.B. Language and cognition in bilinguals and multilinguals: An introduction. United Kingdom: Psychology Press, 2011.

Grosjean, François (a). Life with two languages: An introduction to bilingualism. Harvard University Press, 1982.

Grosjean, François (b). Bilingual: Life and Reality. Harvard: Harvard University Press, 2010.

Hamers, Josiane F.; Blanc, Michel H. A. Bilinguality and bilingualism. Cambridge: Cambridge University Press, 2000.

Heredia, Roberto R.; Brown, Jeffrey M. "Bilingual Memory". The Handbook of Bilingualism, Bhatia, Tej K.; Ritchie, William C. (Eds.). New Jersey: Blackwell Publishing, 2004.

Hernandez, Arturo E. The bilingual brain. Oxford: Oxford University Press, 2013.

Hilchey, Matthew D.; Klein, Raymond M. "Are there bilingual advantages on nonlinguistic interference tasks? Implications for the plasticity of executive control processes". Psychonomic bulletin \& review. 18.4, (2011): 625-658. 
Hoshino, Noriko; Thierry, Guillaume. "Do Spanish-English bilinguals have their fingers in two pies-or is it their toes? An electrophysiological investigation of semantic access in bilinguals". Frontiers in psychology. 3, (2012): 9.

Hosoda, Chihiro; et al. "Dynamic neural network reorganization associated with second language vocabulary acquisition: A multimodal imaging study". Journal of Neuroscience. 33.34, (2013): 13663-13672.

Hsieh, Ming-Che; et al. "Neural correlates of bilingual language control during interlingual homograph processing in a logogram writing system". Brain and language. 174, (2017): 72-85.

Jessner, Ulrike. "A DST model of multilingualism and the role of metalinguistic awareness". The modern language journal. 92.2, (2008): 270-283.

Jouravlev, Olessia; Lupker, Stephen J.; Jared, Debra. “Cross-language phonological activation: Evidence from masked onset priming and ERPs". Brain and language. 134, (2014): 11-22.

Kemmerer, David. Cognitive neuroscience of language. New Jersey: Psychology Press, 2014.

Kerkhofs, Roel; et al. "Testing a model for bilingual semantic priming with interlingual homographs: RT and N400 effects". Brain research. 1068.1, (2006): 170-183.

Kousaie, Shanna; Phillips, Natalie A. "Age-related differences in interlingual priming: A behavioural and electrophysiological investigation". Aging, Neuropsychology, and Cognition. 18.1, (2010): 22-55.

Kutas, Marta; Federmeier, Kara D. "Thirty years and counting: finding meaning in the N400 component of the event-related brain potential (ERP)". Annual review of psychology. 62, (2011): 621-647.

Kutas, Marta; Van Petten, Cyma K.; Kluender, Robert. "Psycholinguistics electrified II (1994-2005)". Handbook of psycholinguistics. Amsterdam: Elsevier 2006, pp. 659-724. 
Kroll, Judith F.; Bobb, Susan C.; Hoshino, Noriko. "Two languages in mind: Bilingualism as a tool to investigate language, cognition, and the brain". Current directions in psychological science. 23.3, (2014): 159-163.

Kroll, Judith F.; Dussias, Paola E. "The comprehension of words and sentences in two languages". The handbook of bilingualism. New Jersey: Blackwell Publishing Ltd, 2004, pp. 169-200.

Lemhöfer, Kristin; Dijkstra, Ton. "Recognizing cognates and interlingual homographs: Effects of code similarity in language-specific and generalized lexical decision”. Memory \& Cognition. 32.4, (2004): 533-550.

Lemhöfer, Kristin; Dijkstra, Ton; Michel, Marije. "Three languages, one ECHO: Cognate effects in trilingual word recognition". Language and cognitive processes. 19.5, (2004): 585-611.

Lewis, M. Paul; Simons, Gary F. ; Fennig, Charles D. Ethnologue: Languages of the world. Dallas, Texas: SIL International. 2016.

Libben, Gary. "Disorders of lexis". The Handbook of the neuroscience of language, Stemmer, Brigitte; Whitaker, Harry A. (Eds.). Cambrige: Academic Press. 2008, pp. 147-154.

Luk, Gigi; De Sa, Eric; Bialystok, Ellen. "Is there a relation between onset age of bilingualism and enhancement of cognitive control?". Bilingualism: Language and cognition. 14.4, (2011): 588-595.

Luk, Gigi; Bialystok, Ellen. "Bilingualism is not a categorical variable: Interaction between language proficiency and usage". Journal of Cognitive Psychology. 25.5, (2013): 605-621.

Luk, Gigi; et al. "Lifelong bilingualism maintains white matter integrity in older adults". Journal of Neuroscience. 31.46, (2011): 16808-16813.

Macizo, Pedro; Bajo, Teresa; Martín, María Cruz. "Inhibitory processes in bilingual language comprehension: Evidence from Spanish-English interlexical homographs". Journal of Memory and Language. 63.2, (2010): 232-244. 
Marian, Viorica; Shook, Anthony. "The cognitive benefits of being bilingual". Cerebrum: the Dana forum on brain science. Vol. 2012. Dana Foundation, 2012.

Martin-Rhee, Michelle M.; Bialystok, Ellen. "The development of two types of inhibitory control in monolingual and bilingual children". Bilingualism: language and cognition. 11.1, (2008): 81-93.

Megale, Antonieta Heyden. "Bilinguismo e educação bilíngue-discutindo conceitos”. Revista Virtual de Estudos da Linguagem-ReVEL. 3.5, (2005): 1-13.

Miyake, Akira; et al. "The unity and diversity of executive functions and their contributions to complex 'frontal lobe' tasks: A latent variable analysis". Cognitive psychology. 41.1, (2000): 49-100.

Mohades, Seyede Ghazal; et al. "Age of second language acquisition affects nonverbal conflict processing in children: an fMRI study". Brain and Behavior. 4.5, (2014): 626-642.

Mor, Billy; Yitzhaki-Amsalem, Sarin; Prior, Anat. "The joint effect of bilingualism and ADHD on executive functions". Journal of attention disorders. 19.6, (2015): 527-541.

Oliveira, Gilvan Müller. "Brasileiro fala português: monolinguismo e preconceito linguístico". Revista Linguasagem. 11.1, (2009): 1-9.

Paap, Kenneth R.; Greenberg, Zachary I. "There is no coherent evidence for a bilingual advantage in executive processing". Cognitive psychology. 66.2, (2013): 232-258.

Paap, Kenneth R.; Sawi, Oliver. "Bilingual advantages in executive functioning: problems in convergent validity, discriminant validity, and the identification of the theoretical constructs”. Frontiers in psychology. 5, (2014): 1-15.

Paap, Kenneth R.; Johnson, Hunter A.; Sawi, Oliver. "Are bilingual advantages dependent upon specific tasks or specific bilingual experiences?". Journal of Cognitive Psychology. 26.6, (2014): 615-639. 
Perani, Daniela; Abutalebi, Jubin. "Bilingualism, dementia, cognitive and neural reserve". Current opinion in neurology. 28.6, (2015): 618-625.

Pliatsikas, Christos; Moschopoulou, Elisavet; Saddy, James Douglas. "The effects of bilingualism on the white matter structure of the brain". Proceedings of the National Academy of Sciences. 112.5, (2015): 1334-1337.

Poarch, Gregory J.; Bialystok, Ellen. "Bilingualism as a model for multitasking". Developmental Review. 1.35, (2015): 113-124.

Poarch, Gregory J.; Van Hell, Janet G. "Executive functions and inhibitory control in multilingual children: Evidence from second-language learners, bilinguals, and trilinguals". Journal of experimental child psychology. 113.4, (2012): 535-551.

Poulin-Dubois, Diane; et al. "The effects of bilingualism on toddlers' executive functioning”. Journal of experimental child psychology. 108.3, (2011): 567-579.

Preuss, Elena Ortiz; Álvares, Margarida Rosa. "Bilinguismo e políticas linguísticas no Brasil: da ilusão monolíngue à realidade plurilíngue". Acta Scientiarum. Language and Culture. 36.4, (2014): 403-414.

Prior, Anat; MacWhinney, Brian. "A bilingual advantage in task switching". Bilingualism: Language and cognition. 13.2, (2010): 253-262.

Rodriguez-Fornells, Antoni; et al. "Brain potential and functional MRI evidence for how to handle two languages with one brain". Nature. 415.6875, (2002): 1026-1029.

Schwartz, Ana I.; Fontes, Ana B. Areas da Luz. "Cross-language mediated priming: Effects of context and lexical relationship". Bilingualism: Language and cognition. 11.1, (2008): 95-110.

Singh, Niharika; Mishra, Ramesh K. "The modulatory role of second language proficiency on performance monitoring: evidence from a saccadic countermanding task in high and low proficient bilinguals". Frontiers in psychology. 5.1418, (2015): 1-14. 
Stern, Yaakov (a). "What is cognitive reserve? Theory and research application of the reserve concept". Journal of the international neuropsychological society. 8.3, (2002): 448-460.

Stern, Yaakov (b). "Cognitive reserve”. Neuropsychologia. 47.10, (2009): 20152028.

Tao, Lily; Taft, Marcus; Gollan, Tamar H. "The bilingual switching advantage: Sometimes related to bilingual proficiency, sometimes not". Journal of the International Neuropsychological Society. 21.7, (2015): 531-544.

Thierry, Guillaume; Wu, Yan Jing (a). "Electrophysiological evidence for language interference in late bilinguals". NeuroReport. 15.10, (2004): 1555-1558.

Thierry, Guillaume; Wu, Yan Jing (b). "Brain potentials reveal unconscious translation during foreign-language comprehension". Proceedings of the National Academy of Sciences. 104.30, (2007): 12530-12535.

Van Heuven, Walter JB; et al. "Language conflict in the bilingual brain". Cerebral cortex. 18.11, (2008): 2706-2716.

Van Wijnendaele, Ilse; Brysbaert, Marc. "Visual word recognition in bilinguals: phonological priming from the second to the first language". Journal of Experimental Psychology: Human Perception and Performance. 19.3, (2002): 616-627.

Vega-Mendoza, Mariana; et al. "The impact of late, non-balanced bilingualism on cognitive performance". Cognition. 137, (2015): 40-46.

Von Studnitz, Roswitha E.; Green, David W. "Interlingual homograph interference in German-English bilinguals: Its modulation and locus of control". Bilingualism: Language and Cognition. 5.1, (2002): 1-23.

Wong, Becky; Yin, Bin; O’Brien, Beth. "Neurolinguistics: structure, function, and connectivity in the bilingual brain". BioMed research international. 2016. 
Woumans, E. V. Y.; et al. "Bilingualism delays clinical manifestation of Alzheimer's disease”. Bilingualism: Language and Cognition. 18.3, (2015): 568-574.

Recebido em: 28/08/2020

Aceito em: 11/11/2020

Publicado em dezembro de 2020

Marina Fernandes Neves Lameira. E-mail: lameira.marina@gmail.com. ORCID: https://orcid.org/0000-0003-1937-3408

Elaine Cristina de Barros Torresi. E-mail: ectorresi@hotmail.com. ORCID: https://orcid.org/0000-0002-3106-8855

Katerina Lukasova. E-mail: katerinaluka@gmail.com. ORCID: https://orcid. org/0000-0002-1137-7298

Maria Teresa Carthery-Goulart. E-mail: teresa.carthery@ufabc.edu.br. ORCID: https://orcid.org/0000-0002-2751-4541

Cad. Trad., Florianópolis, v. 40, $\mathrm{n}^{0}$ esp. 2, p. 185-216, set-dez, 2020.216 